\title{
Lethal Mutations and Balanced Lethal Systems in Aspergillus nidulans
}

\author{
By J. L. AZEVEDO* AND J. A. ROPER \\ Department of Genetics, University of Sheffield
}

(Accepted for publication 28 April 1967)

SUMMARY

The frequency of spontaneous and ultraviolet-induced, recessive, lethal mutations was estimated in two diploid strains of Aspergillus nidulans. The parent diploids were heterozygous for recessive and semi-dominant markers; each recessive lethal resulted in failure to recover haploid strains carrying the allele linked in coupling to that lethal. Diploids I and 2 carried markers permitting direct assay of lethals on 4 and 8 chromosomes, respectively, out of the total diploid complement of 16 . No lethals were detected in 100 isolates from each untreated diploid. One hundred isolates from each treated parent yielded 6 and 16 lethals, respectively, suggesting a frequency for the whole genome, at $5 \%$ survival, of about $28 \%$ lethals. The lethals were nutritionally irreparable and were not temperature-sensitive. Several of them have been located roughly by mitotic crossing-over.

One isolate was heterozygous for two unlinked lethals. It segregated to give a stable heterokaryon bearing two classes of non-viable haploid conidia. Meiotic analysis, via a three-component heterokaryon, showed that the stable heterokaryon was balanced by the non-allelic lethals, one in each haploid component. Another isolate had a balanced lethal system resulting from linked, non-allelic lethals in trans.

Six isolates from treatment were stable diploids which produced no haploid sectors.

\section{INTRODUCTION}

Recessive lethal mutations have been used extensively in higher organisms as objective measures of mutation rates, as a tool in developmental genetics and as a means of maintaining balanced lethal systems. Most micro-organisms have haploid nuclei and this has restricted the study of irreparable mutations except in the case of temperature-sensitive or other environment-dependent lethal mutants. Atwood (1949) circumvented this difficulty in an elegant fashion through the use of heterokaryotic multinucleate conidia of Neurospora. The conidia of Aspergillus are uninucleate, but the availability of strains with diploid nuclei permits the study of recessive lethals. Tector \& Käfer (1962) and Käfer \& Chen (1964) have already exploited this system. The present work was undertaken to measure the frequency of spontaneous and induced irreparable lethals and to explore the possibility of their use to stabilize heterokaryons and strains with diploid nuclei.

\footnotetext{
* Present address: Institute of Genetics, University of São Paulo, Piracicaba, Brazil.
} 


\section{METHODS}

The general techniques used throughout this work were those of Pontecorvo et al. (1953). Incubation was at $37^{\circ}$ except where otherwise stated.

Media. Minimal medium (MM) was Czapek-Dox medium with $\mathrm{I} \%$ glucose. Complete medium (CM) was a complex medium, containing yeast extract, casein hydrolysate, hydrolysed nucleic acid, vitamins, etc. Solid media contained $2 \%$ agar.

Inhibitors. DL-p-fluorophenylalanine ( $p \mathrm{FA}$; from Koch-Light and Co. Ltd., Poyle Trading Estate, Colnbrook, Buckinghamshire) was used in CM and supplemented MM at 20 and $40 \mu \mathrm{g} . / \mathrm{ml}$., respectively. Acriflavine (British Drug Houses, Ltd., Poole, Dorset) was used at $25 \mu \mathrm{g} . / \mathrm{ml}$.

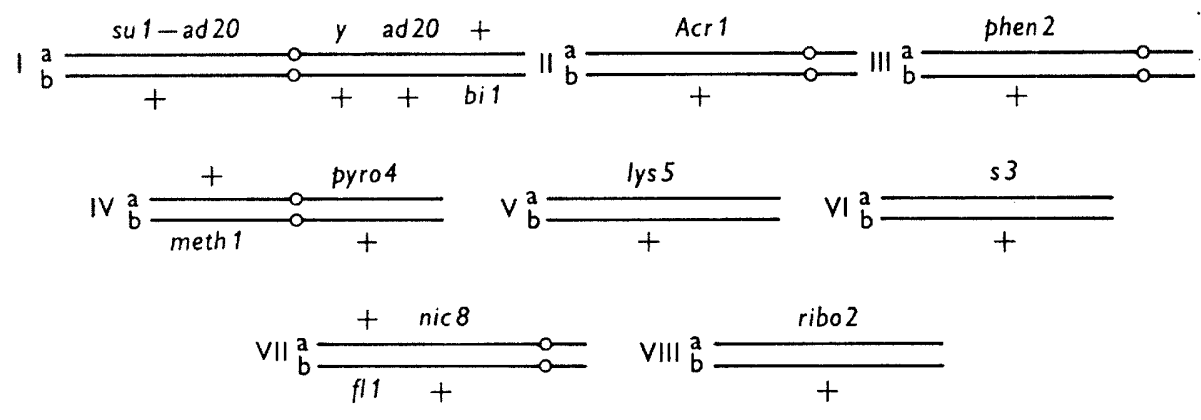

Diploid 1

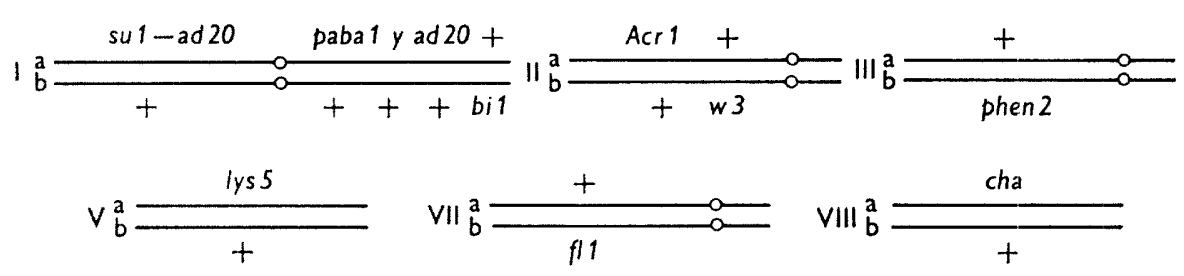

Diploid 2

Fig. I. Aspergillus nidulans. Genotypes of diploids I and 2.

Strains. Genotypes of the two diploid strains used in this work are shown in Fig. I. Mutant alleles, and their locations, were given by Pontecorvo et al. (I953), Käfer (I958), Pontecorvo (1963), Ball \& Azevedo (1964) and Barratt, Johnson \& Ogata (1965). Alleles used in this work were: $y, w 3$, cha determining, respectively, yellow, white, chartreuse conidia; $f l_{I}$, fluffy; ad 20 , biI, lys 5 , methI and meth 2, nic 8, pabaI, phen 2 , pyro 4, ribo 2, $s_{I 2}$ and $s_{3}$ determining, respectively, requirement for adenine, biotin, lysine, methionine, nicotinic acid, $p$-aminobenzoic acid, phenylalanine, pyridoxin, riboflavine, thiosulphate; suI-ad 20, suppressor of ad2o; AcrI, resistance to acriflavine; mal $\mathrm{I}$, inability to use maltose.

Diploids I and 2 were free from chromosomal translocations; they were synthesized by the method of Roper (1952) and kept on MM. 
Ultraviolet (u.v.) irradiation. Saline suspensions of conidia were irradiated by a mercury vapour lamp to give about $5 \%$ survival.

Induction and detection of recessive lethal mutations. Conidia, untreated or irradiated to $5 \%$ survival, were plated on MM and incubated 3-4 days. For each diploid 200 colonies were taken, I00 control and Ioo following treatment; colonies which showed morphological abnormality were excluded. Each such isolate was purified by selection of a single colony from a plating of conidia on MM.

Each isolate was tested for newly arisen recessive mutations by the following steps. First, by plating conidia, about 10 per dish, on $\mathrm{MM}+p \mathrm{FA}+$ all nutritional requirements of the haploid parents. Diploid strains grow poorly on $p F A$ but the haploid nuclei formed during this stunted growth gave vigorous sectors (Morpurgo, 196r ; Lhoas, 196I). Mitotic haploidization does not involve crossing-over (Pontecorvo, Tarr Gloor \& Forbes, 1954); in the absence of a lethal mutation all the markers of the parent haploids, apart from the exception discussed below, are recovered among the haploid segregants. Absence of any allele from among these segregants suggested a lethal linked in coupling with that allele. Scoring was limited to sectors phenotypically distinct from the parent diploids, which were non-fluffy and had green conidia. With this limitation, diploid I isolates were expected to yield yellow sectors, fluffy and non-fluffy. Diploid 2 was expected to yield yellow, white and chartreuse, fluffy and non-fluffy in each case. This permitted scoring of mutations on 3 and 7 chromosomes, respectively. $p \mathrm{FA}$ selects against haploids carrying phen 2 ; a lethal in coupling with phen ${ }^{+}$would give a non-sectoring isolate. This, subject to later reservations, assayed lethals on a further chromosome in each diploid.

The $p \mathrm{FA}$ test on supplemented $\mathrm{MM}$ would record as lethal any newly arisen nutritional requirement not satisfied by the supplements. Furthermore, $p \mathrm{FA}$ might select against haploids carrying certain new but non-lethal mutations and so overestimate the frequency of lethals. Conidia of each isolate, suspected on the $p \mathrm{FA}$ test of carrying a lethal, were plated on CM and incubated at $37^{\circ}$ and $25^{\circ}$. Segregants differing from the parent diploid in morphology or conidial colour were isolated and tested for ploidy by their conidial size (Roper, 1952). Classification of haploids produced at $37^{\circ}$ provided a cross-check with the $p \mathrm{FA}$ selection; haploids produced at $25^{\circ}$ checked the possible temperature sensitivity of each lethal. Diploid segregants arose mainly from mitotic crossing-over; in appropriate cases they were used for an approximate location of the lethal mutants.

Genetic analysis. The techniques were those of Pontecorvo et al. (I953), with a modified technique for ascus dissection (Bainbridge, 1964). The methods of Roper \& Käfer (1957) and Pritchard (1955) were used for the selection of mitotic segregants by resistance to acriflavine and by suppression of adenine requirement, respectively.

\section{RESULTS}

\section{Lethal mutations in diploid I}

One hundred control isolates carried no lethals on the chromosomes tested. In summary, the roo isolates from treatment were as follows: 92 sectored normally in respect of the four tested chromosomes; one isolate carried a mutation, located on chromosome I, which determined requirement for $p$-aminobenzoic acid; three carried single lethals; one carried two unlinked lethals and gave the balanced heterokaryon 
described below; one carried two non-allelic, linked, recessive lethals in trans arrangement; the remaining two isolates failed to sector.

The three isolates carrying single mutations were analysed readily by their sectoring behaviour on $\mathrm{CM}, \mathrm{CM}+p \mathrm{FA}$ and $\mathrm{CM}+$ acriflavine. The two isolates which carried two lethal mutations each required more intensive investigation as they were included initially in the series failing to give any haploid sectors.

One of these isolates carried a lethal in cis arrangement with phen $2^{+}$(chromosome III) and was therefore expected to give no sectors on $p \mathrm{FA}$ media. The other lethal was shown to be carried in cis arrangement with $\mathrm{fl}_{\mathrm{I}^{+}}$(chromosome VII) since all haploid sectors on CM carried phen 2 and $f t I$. In fact the isolate yielded a single sector on $p \mathrm{FA}$. The yellow conidia of this sector, plated on $\mathrm{CM}$ or supplemented MM, gave micro-colonies about $60 \mu$ in diameter but did not grow further. The sector, which had a growth requirement for pyridoxin and riboflavine, could be maintained only by subculture of mycelium. It seemed likely that the sector was a balanced heterokaryon produced by the breakdown of a diploid heterozygous for two non-allelic lethals; the heterokaryon carried one lethal in each of its haploid components, which were designated $A l r$ and $B l 2$. This was confirmed by meiotic analysis. The heterokaryon was combined with the strain meth 2 phen 2 ; nic 8 mal I $f I$ (designated $C$ ) in a three-component heterokaryon maintained in balance on MM. With relatively rare exceptions, each perithecium of Aspergillus nidulans derives from two nuclei only (Hemmons, Pontecorvo \& Bufton, 1953). From a three-component heterokaryon six classes of perithecia are to be expected, three hybrid and three selfed. Five distinguishable classes were found, including all three hybrid classes and the selfed non-lethal class. Some perithecia yielded only non-viable ascospores; since the lethals could not be distinguished on their germination pattern, these perithecia may have included both selfed lethal types. Genotypes of nuclei $A$ and $B$ were determined by analysis of $A \times C$ and $B \times C$ perithecia; they were-A: suI-ad 20 y ad $20 ; l I$; pyro 4 ; flI; ribo 2; and $B$ : su I-ad 2o y ad 2o; AcrI; phen 2; pyro 4; l2 nic 8; ribo2. 12 showed free recombination with mal $I$, nic 8 and $f_{I} ; l_{I}$ was located $I 6 \pm 3$ units from meth 2 , tentatively between meth 2 and phen 2.

The diploid isolate carrying non-allelic lethals in trans also did not sector normally. It produced a single poorly growing sector on $\mathrm{CM}+p \mathrm{FA}$. Plating of the sparse yellow conidia of this sector gave abnormal colonies which, on further incubation, yielded vigorous stable sectors. Both sectors and centres required adenine, pyridoxin and nicotinic acid and were acriflavine-sensitive. The sectors carried conidia of diploid size; conidia from the colony centres were variable in size but mainly near-haploid. The stable diploid sectors, arising from the poorly growing centres, did not sector haploids on $p F A$. The single poorly growing sector was probably an aneuploid which arose following two types of mitotic event: (i) mitotic crossing-over between $y$ and its centromere which gave homozygosis for $y$ and ad 2o; (ii) loss of one member from certain chromosome pairs which gave an aneuploid (Käfer, 196I) disomic for chromosome I, monosomic for II, IV and VII and, perhaps, monosomic for the remaining chromosomes. The aneuploid was unable to produce viable haploid nuclei; by non-disjunction it could produce diploid nuclei which gave stable diploid sectors. The original isolate did not produce diploid suI-ad2o/suI-ad 20 or haploid su I-ad 20 sectors by Pritchard's (I955) technique and this implied a lethal distal to and in cis arrangement with suI-adzo. It seemed probable that the isolate, and the 
aneuploid derived from it, carried non-allelic lethals in trans on chromosome I. The lethal on Ib is excluded from calculations on the total lethal frequency since chromosome Ib was not generally assayed.

\section{Lethal mutations in diploid 2}

One hundred control untreated isolates sectored normally on $p \mathrm{FA}$. Treatment gave 80 isolates with no lethals on the 8 chromosomes tested, and 16 with one lethal each. The remaining 4 isolates, still diploid, produced no haploid sectors on any media. The 16 isolates carrying lethals sectored on $p \mathrm{FA}$ and permitted chromosomal location of all the lethals.

Table 1. Aspergillus nidulans. Chromosomal location of ultraviolet-induced lethals

$\begin{array}{lcr} & \text { Chromosome* } & \text { Number o } \\ \text { Diploid I } & \text { Ia } & 3 \\ & \text { IIIb } & 2 \\ \text { VIIa } & \text { I } \\ \text { VIIb } & 0 \\ \text { Diploid 2 } & \text { Ia } & 3 \\ & \text { IIa } & 2 \\ \text { II b } & 3 \\ \text { IIIa } & 4 \\ \text { VIIa } & 0 \\ \text { VIIb } & 3 \\ \text { VIIIa } & 1 \\ \text { VIIIb } & 0\end{array}$

* See Fig. 1 .

Table 2. Aspergillus nidulans. Decreased frequency of certain diploid segregants from lethal-bearing isolates of diploid 2

The number of diploid segregants was estimated from 44 point inoculations of each strain on CM.

\begin{tabular}{|c|c|c|c|c|c|c|}
\hline \multirow[b]{2}{*}{ Isolate } & & \multicolumn{4}{|c|}{ Segregants } & \multirow[b]{2}{*}{$\begin{array}{c}\text { Implied } \\
\text { location of } \\
\text { lethal }\end{array}$} \\
\hline & $\begin{array}{c}\text { Chromosomal } \\
\text { location of } \\
\text { lethal }\end{array}$ & \multicolumn{4}{|c|}{ Class and number } & \\
\hline 118 & II b & 20 & $10^{*}$ & 32 & 9 & Proximal to $w 3$ \\
\hline 119 & II b & 19 & $0^{*}$ & 30 & 8 & Distal to $w_{3}$ \\
\hline 143 & VIIb & 25 & 38 & $0^{*}$ & I I & Distal to $f l I$ \\
\hline 179 & VII b & 25 & 25 & $I^{*}$ & I I & Proximal to $f l I$ \\
\hline 196 & Ia & $8 * \dagger$ & 37 & 34 & 20 & $\begin{array}{l}\text { Between paba and } \\
\text { centromere }\end{array}$ \\
\hline Control & - & 22 & 33 & 30 & 14 & \\
\hline
\end{tabular}

\section{Location of lethals}

Each lethal was located to a particular chromosome, mainly on the basis of haploidization (Table I). More precise location of some lethals induced in diploid I has been described above. 
It was sometimes possible to attempt more precise location of a lethal by estimating the frequency with which a lethal-bearing isolate produced certain classes of diploid segregants by mitotic crossing-over. For example, an isolate with a lethal distal to $y$ and in cis arrangement would produce no homozygous $y$ sectors; a lethal proximal to $y$ would decrease the frequency of such sectors; a lethal on the left arm of I should not affect the frequency of diploid yellow sectors. In appropriate cases the frequency of diploid sectors produced by lethal-bearing isolates was compared with the frequency of such sectors from the diploid parent. The significant results are summarized in Table 2.

\section{DISCUSSION}

The present study has yielded a total of 22 irreparable lethals following ultraviolet treatment to $5 \%$ survival; 6 came from 100 isolates of a diploid assaying 4 chromosomes and 16 from a diploid assaying 8 chromosomes out of the diploid complement of r6. Similar numbers of isolates from untreated diploids gave no lethals. On the assumption that the frequency of lethals is the same for all chromosomes-an assumption unlikely to be fully justified-the lethal frequency for the whole genome was between $24 \%$ and $32 \%$. This agrees well with the $25 \%$ found by Käfer \& Chen (1964) under similar conditions.

Despite an intensive investigation, 6 of the 200 u.v. isolates gave no haploid sectors, though they did produce diploid segregants resulting from mitotic crossing-over. Two other isolates produced only very rare sectors and they were shown to carry two lethal mutations each. It is possible, then, that the refractory six isolates also carried multiple lethals. Lethal mutations are likely to be genetically heterogenous and to include, for example, translocations of a type permitting no viable haploid segregants. Several of the six isolates produced diploid segregants at a frequency significantly less than the control and this would tend to support the idea of multiple lethals. Käfer (1963) described similar cases of stable diploids and Tector \& Käfer (I962) observed such strains after gamma-irradiation.

Discovery of the parasexual cycle (Pontecorvo \& Roper, 1952) opened the way to the planned breeding of certain asexual fungi used in industrial fermentations. Sermonti (1959), Ishitani, Ikeda \& Sakaguchi (1956), Ikeda, Nakamura, Uchida \& Ishitani (1957) and Macdonald (1964) have discussed the possible value of diploid strains of fungi for industrial purposes, since such diploids would be buffered against selection of undesirable spontaneous recessive mutations; their main disadvantage might lie in the production of haploid segregants and diploid mitotic cross-over types. The present study has shown the relative ease with which balanced lethal systems can be produced and used to maintain stable diploids or heterokaryons. The use of such systems would prevent or decrease the frequency of viable haploid segregants and, in certain cases, would decrease the effective frequency of mitotic crossing-over.

Of the 22 lethal mutants detected, none was temperature-sensitive and the lethals could be maintained only in heterozygous, or in one case heterokaryotic, condition. Haploid conidia, from a heterokaryon with a different lethal in each component, gave micro-colonies about $60 \mu$ in diameter. It would be of interest to know whether lethal mutations in Aspergillus, like those of other organisms, have different times of action (see Hadorn, 195I ; Epstein et al. 1963). This is now being studied in strains carrying temperature-sensitive lethals which permit genetical and biochemical study of the 
mutant lesion in haploid as well as in heterokaryotic and heterozygous diploid condition.

A British Council Fellowship and support for one of us (J.L. A.) from the Brazilian Research Council and Foundation of Assistance to Research of São Paulo State are gratefully acknowledged.

\section{REFERENCES}

ATwoon, K.C. (1949). Detection of physiological mutants in Neurospora without the use of selective media. Biol. Bull. mar. biol. Lab., Woods Hole 97, 254.

BAINBRIDGE, B. W. (1964). Genetic factors affecting the morphology of Aspergillus nidulans. Ph.D. thesis, University of Sheffield.

BALl, C. \& AzEVEDo, J. L. (1964). A fluffy mutant in Aspergillus nidulans. Aspergillus News Letter 5, 9 .

Barratt, R. W., Johnson, G. B. \& OGata, W. N. (1965). Wild type and mutant stocks of Aspergillus nidulans. Genetics 52, 233.

Epstein, R. H., Bolle, A., Steinberg, C. M., Kellenberger, E., Boy de la Tour, E., Chevalley, R., Edgar, R. S., Susman, M., Denhardt, G. H. \& Lielausis, A. (I963). Physiological studies of conditional lethal mutants of bacteriophage T 4 D. Cold Spring Harb. Symp. quant. Biol. 28, 375 .

Hadorn, E. (195I). Developmental action of lethal factors in Drosophila. Adv. Genet. 4, 53.

Hemmons, L. M., Pontecorvo, G. \& Bufton, A. W. J. (1953). Perithecium analysis and relative heterothallism. Adv. Genet. 5 , 194.

IKeda, Y., Nakamura, K., UChida, K. \& Ishitani, C. (1957). Two attempts upon improving an industrial strain of Aspergillus oryzae through somatic recombination and polyploidization. J. gen. appl. Microbiol., Tokyo 3, 93.

IsHTTANI, C., IKEDA, Y. \& SAKAGUCHI, K. (1956). Hereditary variation and genetic recombination in Koji-molds (Aspergillus oryzae and Aspergillus sojae). VI. Genetic recombination in heterozygous diploids. J. gen. appl. Microbiol., Tokyo 2, 401.

KäFER, E. (1958). An 8-chromosome map of Aspergillus nidulans. Adv. Genet. 9, 105.

KÄFER, E. (I96I). The processes of spontaneous recombination in vegetative nuclei of Aspergillus nidulans. Genetics $46, \mathrm{I} 58 \mathrm{I}$.

KÄFER, E. (I963). Radiation effects and mitotic recombination in diploids of Aspergillus nidulans. Genetics 48, 27.

KÄFER, E. \& CHEN, T. L. (1964). Translocations and recessive lethals induced in Aspergillus nidulans by ultraviolet light and gamma rays. Can. J. Genet. Cytol. 6, 254.

LhOAS, P. (196I). Mitotic haploidisation by treatment of Aspergillus niger diploids with p-fluorophenylalanine. Nature, Lond. r9o, 734.

MACDONALD, K. D. (1964). Preservation of the heterozygous diploid condition in industrial microorganisms. Nature, Lond. 204, 404.

MoRPURGo, G. (196I). Somatic segregation induced by $p$-fluorophenylalanine. Aspergillus News Letter 2, 4 .

PonteConvo, G. (1963). Glasgow list of located or partially located mutants of Aspergillus nidulans. Aspergillus News Letter 4, 12.

PonteCoRvo, G. \& Roper, J. A. (1952). Genetic analysis without sexual reproduction by means of polyploidy in Aspergillus nidulans. J. gen. Microbiol. 6, vii.

Pontecorvo, G., Roper, J. A., Hemmons, L. M., Macdonald, K. D. \& Bufton, A. W. J. (1953). The genetics of Aspergillus nidulans. Adv. Genet. 5, 141.

PonteCorvo, G., TARr GlOOR, E. \& Forbes, E. (1954). Analysis of mitotic recombination in Aspergillus nidulans. J. Genet. 52, 226.

PrITCHARD, R.H. (I955). The linear arrangement of a series of alleles of Aspergillus nidulans Heredity 9, 343 .

ROPER, J. A. (I952). Production of heterozygous diploids in filamentous fungi. Experientia 8, I4.

ROPER, J. A. \& KÄFER, E. (1957). Acriflavine resistant mutants of Aspergillus nidulans. J. gen. Micro biol. $\mathbf{x 6}, 660$.

Sermontr, G. (1959). Genetics of penicillin production. Ann. N.Y. Acad. Sci. 81, 950.

TECTOR, M. A. \& KäFER, E. (1962). Radiation-induced chromosomal aberrations and lethals in Aspergillus nidulans. Science, N.Y. 136, 1056. 